\section{Program for computing measures of association in two-way contingency tables}

\section{JAMES ARBUCKLE}

Temple University, Philadelphia, Pennsylvania 19122

Description. The program computes various measures of association for two-way contingency tables. Two of these are estimates of the product-moment correlation under the assumption that the contingency table has been obtained by polychotomizing observations on each of two random variables having a bivariate normal distribution. The first of these is an estimate of the product-moment correlation based on the polychoric series developed by Lancaster and Hamdan (1964). The second is the maximum likelihood estimate developed by Tallis (1962). Both methods yield the usual tetrachoric correlation coefficient in the 2 by 2 case. Several ancillary results are printed out along with the maximum likelihood estimate, including a large sample estimate of its standard error and the likelihood ratio ch-square statistic for testing the hypothesis of bivariate normality. In addition, maximum likelihood estimates of the category boundaries are printed out after imposing the arbitrary identifiability condition that each random variable have zero mean and unit variance.

Several other measures of association described by Kendall and Stuart (1973, Chapter 33) are also computed. These include (1) Pearson's chi-square statistic for testing independence, (2) Pearson's mean square contingency, (3) Pearson's coefficient of contingency, (4) Tschuprow's coefficient of association, and (5) Cramer's modification of Tschuprow's coefficient. An option is provided for reanalyzing a set of data after reordering or collapsing categories.

Language. The program is written in FORTRAN IV and has been run and tested on the CDC-6400 operating under SCOPE 3.4.1. Only standard FORTRAN features have been employed, and no difficulties in conversion to other operating environments are anticipated.

Computation Time and Space. Running on the CDC -6400 , the analysis of an 8 by 10 table takes about $7.5 \mathrm{sec}$. The program, with dimensioning allowing tables as large as 20 by 20 , occupies about $28 \mathrm{~K}$ of core space.

Availability. A listing of the program together with documentation and sample input and output may be obtained at no cost from the author at the Department of Psychology, Temple University, Philadelphia, Pennsylvania 19122.

\section{REFERENCES}

Kendall, M. G., \& Stuart, A. The advanced theory of statistics (Vol. 2, 3rd ed.). New York: Hafner, 1973.

Lancaster, H. O., \& Hamdan, M. A. Estimation of the correlation coefficient in contingency tables with possibly nonmetrical characters. Psychometrika, 1964, 29, 383-391.

TALLIS, G. $M$. The maximum likelihood estimation of correlation from contingency tables. Biometrics, 1962, 18, 342-353.

(Accepted for publication February 7, 1979.) 